PROCEEDINGS OF THE

AMERICAN MATHEMATICAL SOCIETY

Volume 140, Number 8, August 2012, Pages 2791-2801

S 0002-9939(2011)11188-2

Article electronically published on December 19, 2011

\title{
NONISOTROPIC DILATIONS AND THE METHOD OF ROTATIONS WITH WEIGHT
}

\author{
SHUICHI SATO \\ (Communicated by Michael T. Lacey)
}

\begin{abstract}
We consider maximal functions $M f(x, \theta)$, singular integrals $H f(x, \theta)$, and maximal singular integrals $H_{*} f(x, \theta)$ on $\mathbb{R}^{n} \times S^{n-1}$ associated with homogeneous curves, for functions $f$ on $\mathbb{R}^{n}$. We prove certain weighted mixed norm estimates for them. These results are applied to the theory of singular integrals with variable kernels via the method of rotations of CalderónZygmund.
\end{abstract}

\section{INTRODUCTION}

Let $\left\{A_{t}\right\}_{t>0}$ be a dilation group on $\mathbb{R}^{n}$ defined by $A_{t}=t^{P}=\exp ((\log t) P)$, where $P$ is an $n \times n$ real matrix whose eigenvalues have positive real parts. We assume $n \geq 2$. We can define a norm function $r$ on $\mathbb{R}^{n}$ from $\left\{A_{t}\right\}_{t>0}$ such that:

(1) $r(x) \geq 0, r(x)=r(-x)$ for all $x \in \mathbb{R}^{n}$, and $r(x)=0$ if and only if $x=0$;

(2) $r$ is continuous on $\mathbb{R}^{n}$ and infinitely differentiable in $\mathbb{R}^{n} \backslash\{0\}$;

(3) $r\left(A_{t} x\right)=\operatorname{tr}(x)$ for all $t>0$ and $x \in \mathbb{R}^{n}$;

(4) $r(x+y) \leq C(r(x)+r(y))$ for some $C>0$;

(5) $\Sigma=\left\{\theta \in \mathbb{R}^{n}:\langle B \theta, \theta\rangle=1\right\}$ for a positive symmetric matrix $B$, where $\Sigma=\left\{x \in \mathbb{R}^{n}: r(x)=1\right\}$ and $\langle\cdot, \cdot\rangle$ denotes the Euclidean inner product in $\mathbb{R}^{n}$;

(6) $d x=t^{\gamma-1} d \mu d t$, that is,

$$
\int_{\mathbb{R}^{n}} f(x) d x=\int_{0}^{\infty} \int_{\Sigma} f\left(A_{t} \theta\right) t^{\gamma-1} d \mu(\theta) d t, \quad d \mu=\omega d \mu_{0},
$$

where $\omega$ is a strictly positive $C^{\infty}$ function on $\Sigma, d \mu_{0}$ is the Lebesgue surface measure on $\Sigma$ and $\gamma=\operatorname{trace} P$;

(7) there are positive constants $c_{1}, c_{2}, c_{3}, c_{4}, \alpha_{1}, \alpha_{2}, \beta_{1}$ and $\beta_{2}$ such that

$$
\begin{gathered}
c_{1}|x|^{\alpha_{1}}<r(x)<c_{2}|x|^{\alpha_{2}} \quad \text { if } r(x) \geq 1, \\
c_{3}|x|^{\beta_{1}}<r(x)<c_{4}|x|^{\beta_{2}} \quad \text { if } 0<r(x) \leq 1 .
\end{gathered}
$$

See 2, 18, 22, for more details.

Let $A^{*}$ denote the adjoint of a matrix $A$. Then $A_{t}^{*}=\exp \left((\log t) P^{*}\right)$. We define a norm function $s$ from $\left\{A_{t}^{*}\right\}$ in the same way as we define $r$ from $\left\{A_{t}\right\}$. The function $s$ has properties similar to those of $r$.

Received by the editors November 9, 2010 and, in revised form, March 14, 2011.

2010 Mathematics Subject Classification. Primary 42B20, 42B25.

Key words and phrases. Singular integrals, homogeneous curves, nonisotropic dilation, method of rotations. 
For $t<0$, define $A_{t}$ by $A_{t}=(\operatorname{sgn} t) A_{|t|}=-A_{|t|}$. Let $S^{n-1}$ denote the unit sphere of $\mathbb{R}^{n}$. For $(x, \theta) \in \mathbb{R}^{n} \times S^{n-1}$, we define

$$
\begin{gathered}
M f(x, \theta)=\sup _{h>0} h^{-1}\left|\int_{0}^{h} f\left(x-A_{t} \theta\right) d t\right|, \\
H f(x, \theta)=\text { p.v. } \int_{-\infty}^{\infty} f\left(x-A_{t} \theta\right) d t / t, \\
H_{*} f(x, \theta)=\sup _{0<\epsilon<R}\left|\int_{\epsilon<|t|<R} f\left(x-A_{t} \theta\right) d t / t\right| .
\end{gathered}
$$

Let $w$ be a weight function. We recall that

$$
\|F\|_{L_{w}^{p}\left(L^{q}\right)}=\left(\int_{\mathbb{R}^{n}}\left(\int_{S^{n-1}}|F(x, \theta)|^{q} d \sigma(\theta)\right)^{p / q} w(x) d x\right)^{1 / p}
$$

for functions $F \in L_{w}^{p}\left(L^{q}\left(S^{n-1}\right)\right)$, with usual modifications when $q=\infty$ or $p=\infty$, where $d \sigma$ denotes the Lebesgue surface measure on $S^{n-1}$. Also, we write $\|f\|_{L_{w}^{p}}=$ $\left\|f w^{1 / p}\right\|_{L^{p}}=\left\|f w^{1 / p}\right\|_{p}$ for $f \in L_{w}^{p}\left(\mathbb{R}^{n}\right)$.

For $1 \leq p, q \leq \infty$, let $\Delta_{(p, q)} \subset[0,1] \times[0,1]$ be the interior of the convex hull of the points $(0,0),(1,1),(0,1),(1 / p, 1 / q)$. Put $q_{n}(p)=p(n-1) /(n-p), p_{n}=$ $\max (2,(n+1) / 2)$, where we define $q_{n}(p)=\infty$ when $p \geq n$. Then the following result was proved in [8] (see [14] for earlier work).

Theorem A. Suppose $P=E$ (the identity matrix). Let $(1 / p, 1 / q) \in \Delta_{\left(p_{n}, q_{n}\left(p_{n}\right)\right)}$. Then, $M, H$ and $H_{*}$ are bounded from $L^{p}\left(\mathbb{R}^{n}\right)$ to $L^{p}\left(L^{q}\right)$.

This result for $M, H$ was extended to the case of nonisotropic dilations by [1] as follows.

Theorem B. Let $M$ and $H$ be as in (1.1) and (1.2). Then:

(1) If $(1 / p, 1 / q) \in \Delta_{\left(p_{n}, q_{n}\left(p_{n}\right)\right)}, M$ is bounded from $L^{p}\left(\mathbb{R}^{n}\right)$ to $L^{p}\left(L^{q}\right)$;

(2) the operator $H$ is bounded from $L^{p}\left(\mathbb{R}^{n}\right)$ to $L^{p}\left(L^{q}\right)$ whenever $(1 / p, 1 / q) \in$ $\Delta_{\left(2, q_{n}(2)\right)}$.

See [5, 6, 20, for previous results. To prove Theorem B, Bez [1] showed certain $L^{2}\left(S^{n-1}\right)$ estimates for trigonometric integrals (see Lemma 2 below) by using the decay estimates for the Fourier transform of $d \sigma$. Further, to prove part (1) for $p>2$, [1] found that a result of [15] (see also [16]) can be applied; at the corresponding point in the isotropic case of [8], a result for X-ray transforms of [7] was used.

In this note we shall prove an analogue of Theorem B (2) for the maximal singular integral operator $H_{*}$. We assume that $\Sigma=S^{n-1}, d \mu=\omega d \sigma$ and $\omega$ is even, where $\Sigma, d \mu, \omega$ are as in statements (5), (6) above (see [2, 11, 18, for relevant results).

Theorem 1. Let $H_{*}$ be as in (1.3). Suppose that $(1 / p, 1 / q) \in \Delta_{\left(2, q_{n}(2)\right)}$. Then $H_{*}$ is bounded from $L^{p}\left(\mathbb{R}^{n}\right)$ to $L^{p}\left(L^{q}\right)$.

We recall the following result for $H_{*}$ shown by Chen [5].

Theorem C. Suppose $n=2$ and $P=\operatorname{diag}\left(\alpha_{1}, \alpha_{2}\right)$ with $1<\alpha_{2} / \alpha_{1}<4 / 3$. Then $H_{*}$ is bounded from $L^{p}\left(\mathbb{R}^{2}\right)$ to $L^{p}\left(L^{q}\left(S^{1}\right)\right)$ whenever $(1 / p, 1 / q) \in \Delta_{(2,4)}$. 
Theorem 1 improves on Theorem $\mathrm{C}$ when $n=2$. It is known that if $H$ is bounded from $L^{p}$ to $L^{p}\left(L^{q}\right), p \in(1, \infty)$, then $q \leq q_{n}(p)$ (see Theorem 6 (1) of [1]). This implies the same result for $H_{*}$. Thus, in particular, we can see that Theorem 1 is a sharp result when $n=2$ (we note that $\left.\Delta_{\left(2, q_{2}(2)\right)}=\Delta_{(2, \infty)}\right)$.

If $B$ is a subset of $\mathbb{R}^{n}$ such that $B=\left\{x \in \mathbb{R}^{n}: r(x-a)<t\right\}$ for some $a \in \mathbb{R}^{n}$ and $t>0$, then we call $B$ an $r$-ball. Let $w$ be a weight function on $\mathbb{R}^{n}$. For $1 \leq p<\infty$, we recall the Muckenhoupt class $\mathcal{A}_{p}$. We say $w \in \mathcal{A}_{p}, 1<p<\infty$, if

$$
\sup _{B}\left(|B|^{-1} \int_{B} w(x) d x\right)\left(|B|^{-1} \int_{B} w(x)^{-1 /(p-1)} d x\right)^{p-1}<\infty
$$

where the supremum is taken over all $r$-balls $B$. The class $\mathcal{A}_{1}$ is defined to be the set of weight functions $w$ satisfying $M_{H L} w \leq C w$ a.e., where $M_{H L}$ is the HardyLittlewood maximal operator defined by

$$
M_{H L} g(x)=\sup _{t>0} t^{-\gamma} \int_{r(x-y)<t}|g(y)| d y .
$$

We note that $\mathcal{A}_{p} \subset \mathcal{A}_{u}$ if $p \leq u$. We shall prove the following weighted estimates.

Theorem 2. Let $2 \leq q<q_{n}(2)$. Then, $M, H, H_{*}$ are bounded from $L_{w}^{2}\left(\mathbb{R}^{n}\right)$ to $L_{w}^{2}\left(L^{q}\right)$ for $w \in \mathcal{A}_{1}^{\tau}, \tau=2(n-1) / q-n+2$, where $\mathcal{A}_{1}^{\tau}$ is a subclass of $\mathcal{A}_{1}$ defined by $\mathcal{A}_{1}^{\tau}=\left\{v^{\tau}: v \in \mathcal{A}_{1}\right\}$.

The weight class $\mathcal{A}_{1}^{\tau}$ is required by our methods using interpolation with a change of measure. By [22] we know that the operator $H_{*}$ is bounded from $L^{p}\left(\mathbb{R}^{n}\right)$ to $L^{p}\left(L^{p}\right), 1<p<\infty$, so Theorem 1 follows by interpolation from the part of Theorem 2 concerning $H_{*}$ with $w=1$. By Theorem 2 with $q=2$ and the extrapolation theorem of Rubio de Francia, the operators $M, H, H_{*}$ are bounded from $L_{w}^{p}\left(\mathbb{R}^{n}\right)$ to $L_{w}^{p}\left(L^{2}\right)$ for $w \in \mathcal{A}_{p / 2}, p \geq 2$. Using this and known estimates, we can obtain some other results not explicitly stated in Theorem 2 . For example, we can prove some weighted estimates for $M$ by interpolation with the $L^{p_{n}}-L^{p_{n}}\left(L^{q}\right)\left(q<q_{n}\left(p_{n}\right)\right)$ estimates in Theorem B (1). At present we do not know whether we can get better results by applying in the weighted norm cases the arguments due to [1] of Theorem B (1) for $(1 / p, 1 / q)$ near $\left(1 / p_{n}, 1 / q_{n}\left(p_{n}\right)\right)$.

In Section 2, we shall prove $L_{w}^{2}-L_{w}^{2}\left(L^{q}\right)$ estimates for $M$ of Theorem 2. As in [8], we apply the Plancherel theorem for functions with values in the Sobolev space $L_{\alpha}^{2}\left(S^{n-1}\right)$ and the Littlewood-Paley theory. We prove certain orthogonality estimates (see Lemma 1 below), from which we deduce results for $L^{q}\left(S^{n-1}\right)$ valued functions via the Sobolev embedding theorem (see (2.8)). Also, we prove certain weighted estimates (see $(2.7)$ ). Interpolation with a change of measure between these estimates will readily imply the result of Theorem 2 for $M$.

In Sections 3 and $4, L_{w}^{2}-L_{w}^{2}\left(L^{q}\right)$ estimates of Theorem 2 will be proved for $H$ and $H_{*}$, respectively. We again need the Littlewood-Paley theory and orthogonality estimates for vector valued functions along with interpolation arguments. To handle $H_{*}$, we apply a method of [10] designed to treat certain maximal operators, where the results for $M, H$ obtained in Sections 2 and 3 will be used. We note that the maximal operator $M$ cannot be used to control $H, H_{*}$ as in Lemma 4.1 of [8]; a reason for this is that weighted inequalities which will be required in the arguments are not yet available in the case of nonisotropic dilations.

Finally, in Section 5, we consider singular integrals and maximal singular integrals with variable kernels and we shall prove certain $L^{p}$ and $L_{w}^{p}$ boundedness of 
them (Theorems 3 and 4) by applying Theorems 1 and 2 via the Calderón-Zygmund method of rotations (see [3]). Theorem 3 is an extension to the case of nonisotropic dilations of a result due to $[9$ for isotropic dilation.

\section{2. $L_{w}^{2}\left(L^{q}\right)$ ESTIMATES FOR MAXIMAL FUNCTIONS}

In this section we prove

$$
\|M f\|_{L_{w}^{2}\left(L^{q}\right)} \leq C\|f\|_{L_{w}^{2}}, \quad 2 \leq q<q_{n}(2), \quad f \in \mathcal{S}\left(\mathbb{R}^{n}\right),
$$

where $\mathcal{S}\left(\mathbb{R}^{n}\right)$ denotes the Schwartz class, and $q$ and $w$ are related as in Theorem 2 .

Define $f_{t}(x)=t^{-\gamma} f\left(A_{t}^{-1} x\right), t>0$. We denote by $\hat{f}$ the Fourier transform of $f$ :

$$
\hat{f}(\xi)=\int_{\mathbb{R}^{n}} f(x) e^{-2 \pi i\langle x, \xi\rangle} d x .
$$

Let $\left\{D_{k}\right\}_{-\infty}^{\infty}$ be a sequence of nonnegative functions in $C^{\infty}((0, \infty))$ such that

$$
\begin{gathered}
\operatorname{supp}\left(D_{k}\right) \subset\left[2^{-k-1}, 2^{-k+1}\right], \quad \sum_{k} D_{k}(t)^{2}=1, \\
\left|(d / d t)^{m} D_{k}(t)\right| \leq c_{m} / t^{m} \quad(m=1,2, \ldots) .
\end{gathered}
$$

To apply the Littlewood-Paley theory, we define $S_{k}$ by

$$
\left(S_{k}(f)\right)^{\wedge}(\xi)=D_{k}(s(\xi)) \hat{f}(\xi), \quad k \in \mathbb{Z},
$$

where $\mathbb{Z}$ denotes the set of integers. For $k \in \mathbb{Z}$, let

$$
N_{k} f(x, \theta)=\int_{-\infty}^{\infty} f\left(x-A_{t} \theta\right) \varphi_{k}(t) d t-\int_{\mathbb{R}^{n}} f(x-y) \Phi_{2^{k}}(y) d y,
$$

where $\varphi \in C_{0}^{\infty}(\mathbb{R}), \varphi \geq 0, \operatorname{supp}(\varphi) \subset(1 / 2,2), \int \varphi d t=1, \varphi_{k}(t)=2^{-k} \varphi\left(2^{-k} t\right)$, and $\Phi \in C_{0}^{\infty}\left(\mathbb{R}^{n}\right), \int \Phi d x=1$. Put $\tilde{S}_{k}=S_{k}^{2}$. Then, $\sum_{k} \tilde{S}_{k} f=f$. To prove (2.1), we may assume $f \geq 0$. We note that

$$
\begin{aligned}
M f(x, \theta) & \leq C \sup _{k \in \mathbb{Z}} \int_{-\infty}^{\infty} f\left(x-A_{t} \theta\right) \varphi_{k}(t) d t \\
& \leq C \sup _{k}\left|N_{k} f(x, \theta)\right|+C M_{H L} f(x) \\
& \leq C \sum_{j=-\infty}^{\infty}\left(\sum_{k}\left|N_{k} \tilde{S}_{j+k} f(x, \theta)\right|^{q}\right)^{1 / q}+C M_{H L} f(x) .
\end{aligned}
$$

Let $2 \leq q<q_{n}(2)$. Since $q \geq 2$, (2.2) and the Hardy-Littlewood maximal theorem imply

$$
\begin{aligned}
\|M f\|_{L_{w}^{2}\left(L^{q}\right)} & \leq C \sum_{j}\left\|\left(\sum_{k}\left\|N_{k} \tilde{S}_{j+k} f\right\|_{L^{q}\left(S^{n-1}\right)}^{q}\right)^{1 / q}\right\|_{L_{w}^{2}}+C\|f\|_{L_{w}^{2}} \\
& \leq C \sum_{j}\left\|\left(\sum_{k}\left\|N_{k} \tilde{S}_{j+k} f\right\|_{L^{q}\left(S^{n-1}\right)}^{2}\right)^{1 / 2}\right\|_{L_{w}^{2}}+C\|f\|_{L_{w}^{2}},
\end{aligned}
$$

for $w \in \mathcal{A}_{2}$. We prove the following result. 
Lemma 1. If $0 \leq \alpha<1 / 2$, then

$$
\left\|\left(\sum_{k}\left\|N_{k} \tilde{S}_{j+k} f\right\|_{L_{\alpha}^{2}\left(S^{n-1}\right)}^{2}\right)^{1 / 2}\right\|_{2} \leq C 2^{-\epsilon|j|}\|f\|_{2} \quad \text { for some } \epsilon>0 .
$$

Proof. Let $0<a<1 / 2-\alpha$. It suffices to prove

$$
\begin{aligned}
& \int_{\mathbb{R}^{n}}\left\|N_{k} \tilde{S}_{j+k} f(x, \cdot)\right\|_{L_{\alpha}^{2}}^{2} d x \\
& \leq C \int_{\mathbb{R}^{n}}|\hat{f}(\xi)|^{2}\left|D_{k+j}(s(\xi))\right|^{4} \min \left(\left|A_{2^{k}}^{*} \xi\right|,\left|A_{2^{k}}^{*} \xi\right|^{-1}\right)^{2 a} d \xi .
\end{aligned}
$$

Note that $\left(N_{k} f(\cdot, \theta)\right)^{\wedge}(\xi)=\hat{f}(\xi) \Psi\left(A_{2^{k}}^{*} \xi, \theta\right)$, where

$$
\Psi(\xi, \theta)=\Psi_{0}(\xi, \theta)-\hat{\Phi}(\xi), \quad \Psi_{0}(\xi, \theta)=\int_{-\infty}^{\infty} \exp \left(-2 \pi i\left\langle A_{t} \theta, \xi\right\rangle\right) \varphi(t) d t .
$$

Therefore, to obtain (2.4), it suffices to show the pointwise inequality

$$
\|\Psi(\xi, \cdot)\|_{L_{\alpha}^{2}}^{2} \leq C \min \left(|\xi|,|\xi|^{-1}\right)^{2 a} .
$$

If $|\xi| \leq 1$, this is easily obtained, since $\Psi(\xi, \theta)$ is $C^{\infty}$ and vanishes when $\xi=0$. The estimate (2.5) for $|\xi|>1$ follows from the following result of [1] and integration by parts, as in [8].

Lemma 2. Let $0<c_{1}<c_{2}$ and $\xi \in \mathbb{R}^{n},|\xi|>1$. Then

$$
\int_{S^{n-1}}\left|\int_{c_{1}}^{c_{2}} \exp \left(i\left\langle A_{t} \theta, \xi\right\rangle\right) d t\right|^{2} d \sigma(\theta) \leq C_{\delta}|\xi|^{-1+\delta}
$$

for all $\delta>0$.

This follows from Lemma 8 of 1 and an argument including a change of variables.

We can easily see that

$$
\left\|N_{k} f\right\|_{L_{w}^{2}\left(L^{2}\right)} \leq C\|f\|_{L_{M_{H L}(w)}^{2}} .
$$

Therefore, if $w \in \mathcal{A}_{1}$, by the Littlewood-Paley inequality we have

$$
\left\|\left(\sum_{k}\left\|N_{k} \tilde{S}_{j+k} f\right\|_{L^{2}\left(S^{n-1}\right)}^{2}\right)^{1 / 2}\right\|_{L_{w}^{2}}^{2} \leq C \sum_{k}\left\|\tilde{S}_{j+k} f\right\|_{L_{w}^{2}}^{2} \leq C\|f\|_{L_{w}^{2}}^{2} .
$$

If $2 \leq q<q_{n}(2)$, then by the Sobolev embedding theorem we have $L_{\alpha}^{2}\left(S^{n-1}\right) \subset$ $L^{q}\left(S^{n-1}\right)$ for some $\alpha=\alpha(q) \in[0,1 / 2)$. Thus, Lemma 1 implies

$$
\left\|\left(\sum_{k}\left\|N_{k} \tilde{S}_{j+k} f\right\|_{L^{q}\left(S^{n-1}\right)}^{2}\right)^{1 / 2}\right\|_{2} \leq C 2^{-\epsilon|j|}\|f\|_{2}
$$

for some $\epsilon>0$. Interpolating between (2.7) and (2.8) with change of measure, we get

$$
\left\|\left(\sum_{k}\left\|N_{k} \tilde{S}_{j+k} f\right\|_{L^{q}\left(S^{n-1}\right)}^{2}\right)^{1 / 2}\right\|_{L^{2}\left(w^{\tau}\right)} \leq C 2^{-\epsilon|j|}\|f\|_{L^{2}\left(w^{\tau}\right)}
$$


for some $\epsilon>0$, where $q$ and $\tau$ are related as in Theorem 2. The estimate (2.1) follows from (2.9) and (2.3).

Remark 1. Let $0<c_{1}<c_{2}$ and $\eta, \zeta \in \mathbb{R}^{n} \backslash\{0\}$. Then, we have

$$
\left|\int_{c_{1}}^{c_{2}} \exp \left(i\left\langle A_{t} \eta, \zeta\right\rangle\right) d t\right| \leq C|\langle P \eta, \zeta\rangle|^{-1 / d}
$$

for some positive constant $C$ independent of $\eta$ and $\zeta$, where $d$ is the degree of the minimal polynomial of $P$ (see [21, Corollary in Section 4]). We note that this result implies Lemma 2 when $d=1,2$.

\section{3. $L_{w}^{2}\left(L^{q}\right)$ ESTIMATES FOR SINGULAR INTEGRALS}

Let $H$ be as in (1.2). Let $2 \leq q<q_{n}(2)$. In this section we prove

$$
\|H f\|_{L_{w}^{2}\left(L^{q}\right)} \leq C\|f\|_{L_{w}^{2}}, \quad f \in \mathcal{S}\left(\mathbb{R}^{n}\right),
$$

where $q$ and $w$ are related as in Theorem 2 .

Decompose

$$
H f(x, \theta)=\sum_{k=-\infty}^{\infty} H_{k} f(x, \theta), \quad H_{k} f(x, \theta)=\int_{-\infty}^{\infty} f\left(x-A_{t} \theta\right) \psi_{k}(t) d t
$$

where $\psi_{k}(t)=2^{-k} \psi\left(2^{-k} t\right), \psi \in C_{0}^{\infty}(\mathbb{R}), \operatorname{supp}(\psi) \subset\{1 / 2 \leq|t| \leq 2\}, \int \psi(t) d t=0$. We write

$$
H f=\sum_{k} H_{k} f=\sum_{j} U_{j} f, \quad U_{j} f=\sum_{k} H_{k} S_{j+k}^{2} f,
$$

where $S_{j}$ is as in Section 2. Let $0 \leq \alpha<1 / 2$. We prove

$$
\left\|U_{j} f\right\|_{L^{2}\left(L_{\alpha}^{2}\right)} \leq C 2^{-\epsilon|j|}\|f\|_{2}
$$

for some $\epsilon>0$. Then, arguing as in Section 2, from (3.2) we can get

$$
\left\|U_{j} f\right\|_{L^{2}\left(L^{q}\right)} \leq C 2^{-\epsilon|j|}\|f\|_{2}, \quad 2 \leq q<q_{n}(2) .
$$

Let

$$
\tilde{\Psi}(\xi, \theta)=\int_{-\infty}^{\infty} \exp \left(-2 \pi i\left\langle A_{t} \theta, \xi\right\rangle\right) \psi(t) d t .
$$

We have an estimate similar to (2.5) for $\tilde{\Psi}(\xi, \theta)$, and $\left(H_{k} f(\cdot, \theta)\right)^{\wedge}(\xi)=$ $\hat{f}(\xi) \tilde{\Psi}\left(A_{2^{k}}^{*} \xi, \theta\right)$, where the operator $H_{k}$ is as in the previous paragraph. Therefore, by the Littlewood-Paley theory for vector valued functions,

$$
\begin{aligned}
\left\|U_{j} f\right\|_{L^{2}\left(L_{\alpha}^{2}\right)}^{2} & \leq C \sum_{k}\left\|H_{k} S_{j+k} f\right\|_{L^{2}\left(L_{\alpha}^{2}\right)}^{2} \\
& \leq C \sum_{k} \int_{\mathbb{R}^{n}}\left|D_{j+k}(s(\xi)) \hat{f}(\xi)\right|^{2} \min \left(\left|A_{2^{k}}^{*} \xi\right|,\left|A_{2^{k}}^{*} \xi\right|^{-1}\right)^{2 a} d \xi,
\end{aligned}
$$

where $0<a<1 / 2-\alpha$. This implies

$$
\left\|U_{j} f\right\|_{L^{2}\left(L_{\alpha}^{2}\right)}^{2} \leq C 2^{-\epsilon|j|} \sum_{k} \int_{\mathbb{R}^{n}}\left|D_{j+k}(s(\xi)) \hat{f}(\xi)\right|^{2} d \xi \leq C 2^{-\epsilon|j|}\|f\|_{2}^{2}
$$

for some $\epsilon>0$, which proves (3.2). 
If $w \in \mathcal{A}_{1}$, from an estimate similar to (2.6) and the Littlewood-Paley inequality, we have

$$
\begin{aligned}
\left\|U_{j} f\right\|_{L_{w}^{2}\left(L^{2}\right)} & \leq C\left(\sum_{k}\left\|H_{k} S_{j+k} f\right\|_{L_{w}^{2}\left(L^{2}\right)}^{2}\right)^{1 / 2} \\
& \leq C\left(\sum_{k}\left\|S_{j+k} f\right\|_{L_{w}^{2}}^{2}\right)^{1 / 2} \leq C\|f\|_{L_{w}^{2}} .
\end{aligned}
$$

Interpolation between (3.3) and (3.4) implies

$$
\left\|U_{j} f\right\|_{L_{w^{\tau}}^{2}\left(L^{q}\right)} \leq C 2^{-\epsilon|j|}\|f\|_{L_{w^{\tau}}^{2}}
$$

for some $\epsilon>0$, where $q$ and $\tau$ are related as in Theorem 2. Using (3.5) and the triangle inequality, we can obtain (3.1) as follows:

$$
\|H f\|_{L_{w}^{2}\left(L^{q}\right)} \leq \sum_{j}\left\|U_{j} f\right\|_{L_{w}^{2}\left(L^{q}\right)} \leq C \sum_{j} 2^{-\epsilon|j|}\|f\|_{L_{w}^{2}} \leq C\|f\|_{L_{w}^{2}},
$$

where $q$ and $w$ are as in (3.1).

\section{4. $L_{w}^{2}\left(L^{q}\right)$ ESTIMATES FOR MAXIMAL SINGULAR INTEGRALS}

Let $H_{*}$ be as in (1.3). Let $q, w$ be as in Theorem 2 . In this section we prove

$$
\left\|H_{*} f\right\|_{L_{w}^{2}\left(L^{q}\right)} \leq C\|f\|_{L_{w}^{2}}, \quad f \in \mathcal{S}\left(\mathbb{R}^{n}\right) .
$$

To prove (4.1) we use the following.

Lemma 3. Let

$$
H_{* *} f(x, \theta)=\sup _{N \in \mathbb{Z}}\left|\sum_{k=N}^{\infty} H_{k} f(x, \theta)\right|
$$

Then

$$
\left\|H_{* *} f\right\|_{L_{w}^{2}\left(L^{q}\right)} \leq C\|f\|_{L_{w}^{2}}
$$

where $q, w$ are as in (4.1).

We need the following result, for $p \leq q$, to show Lemma 3 .

Lemma 4. Let $1<p<\infty, 1<q \leq \infty, w \in \mathcal{A}_{p}$. For functions $F(x, \theta)$ on $\mathbb{R}^{n} \times S^{n-1}$, define $\left(M_{H L} F\right)(x, \theta)=\left(M_{H L} F(\cdot, \theta)\right)(x)$. Then

$$
\left\|M_{H L} F\right\|_{L_{w}^{p}\left(L^{q}\right)} \leq C\|F\|_{L_{w}^{p}\left(L^{q}\right)} .
$$

Proof. If $p=q$, this is obvious since $M_{H L}$ is bounded on $L_{w}^{p}\left(\mathbb{R}^{n}\right)$. Also, when $q=\infty$, the inequality holds since $M_{H L}$ is a positive operator. The conclusion follows from interpolation between these results when $p \leq q$. The case $p>q$ can be treated by the duality arguments of [13. We omit the details (see also [19]).

Proof of Lemma 3. Let $\hat{Q} \in C_{0}^{\infty}\left(\mathbb{R}^{n}\right), \operatorname{supp}(\hat{Q}) \subset\{s(\xi)<2\}, \hat{Q}(\xi)=1$ if $s(\xi)<1$. Decompose

$$
\sum_{k=N}^{\infty} H_{k} f=Q_{2^{N}} * H f-Q_{2^{N}} * \sum_{k=-\infty}^{N-1} H_{k} f+\left(\delta-Q_{2^{N}}\right) * \sum_{k=N}^{\infty} H_{k} f,
$$

where $\delta$ denotes the delta function and the convolution is taken with respect to the $x$ variable. 
The first term on the right hand side of (4.2) can be handled by (3.1) and Lemma 4 as follows:

$$
\left\|\sup _{N}\left|Q_{2^{N}} * H f\right|\right\|_{L_{w}^{2}\left(L^{q}\right)} \leq C\left\|M_{H L} H f\right\|_{L_{w}^{2}\left(L^{q}\right)} \leq C\|H f\|_{L_{w}^{2}\left(L^{q}\right)} \leq C\|f\|_{L_{w}^{2}} .
$$

Also, by inspection we see that

$$
\sup _{N}\left|Q_{2^{N}} * \sum_{k=-\infty}^{N-1} H_{k} f(x, \theta)\right| \leq C M_{H L} f(x)
$$

with the constant $C$ independent of $\theta$ (see the proof of Theorem $\mathrm{E}$ in [10]). Therefore, the second term on the right hand side of (4.2) can be handled by the weighted norm inequality for the Hardy-Littlewood maximal operator.

It remains to estimate

$$
I(f)=\sup _{N}\left|\left(\delta-Q_{2^{N}}\right) * \sum_{k=N}^{\infty} H_{k} f\right| .
$$

We note that

$$
I(f) \leq \sum_{j=0}^{\infty} I_{j}(f), \quad I_{j}(f)=\sup _{N \in \mathbb{Z}}\left|\left(\delta-Q_{2^{N}}\right) * H_{N+j} f\right| .
$$

Let $0 \leq \alpha<1 / 2$ and $0<a<1 / 2-\alpha$. Then, as in the proof of (3.2), we have

$$
\left\|\left(\delta-Q_{2^{N}}\right) * H_{N+j} f\right\|_{L^{2}\left(L_{\alpha}^{2}\right)}^{2} \leq C \int_{\mathbb{R}^{n}}\left|\left(1-\hat{Q}\left(A_{2^{N}}^{*} \xi\right)\right) \hat{f}(\xi)\right|^{2}\left|A_{2^{N+j}}^{*} \xi\right|^{-2 a} d \xi .
$$

Therefore,

$$
\sum_{N}\left\|\left(\delta-Q_{2^{N}}\right) * H_{N+j} f\right\|_{L^{2}\left(L_{\alpha}^{2}\right)}^{2} \leq C 2^{-j \epsilon}\|f\|_{2}^{2},
$$

and hence, if $2 \leq q<q_{n}(2)$, the Sobolev embedding theorem implies

$$
\sum_{N}\left\|\left(\delta-Q_{2^{N}}\right) * H_{N+j} f\right\|_{L^{2}\left(L^{q}\right)}^{2} \leq C 2^{-j \epsilon}\|f\|_{2}^{2}
$$

for some $\epsilon>0$.

We write $\delta-Q_{2^{N}}=\sum_{m \leq N} \Delta_{m}, \hat{\Delta}_{m}(\xi)=\Gamma\left(A_{2^{m}}^{*} \xi\right)$, where $\Gamma \in C_{0}^{\infty}, \operatorname{supp}(\Gamma) \subset$ $\left\{c_{1}<s(\xi)<c_{2}\right\}$ for some $c_{1}, c_{2}>0$. Then, by Plancherel's theorem we have

$$
\left\|\Delta_{m} * H_{N+j} f\right\|_{L^{2}\left(L^{2}\right)}^{2} \leq C 2^{-\epsilon(N-m+j)}\|f\|_{2}^{2} .
$$

On the other hand, if $w \in \mathcal{A}_{1}$, as in (3.4), we see that

$$
\left\|\Delta_{m} * H_{N+j} f\right\|_{L_{w}^{2}\left(L^{2}\right)}^{2} \leq C\|f\|_{L_{w}^{2}}^{2} .
$$

For $w \in \mathcal{A}_{1}$, choose $b>1$ such that $w^{b} \in \mathcal{A}_{1}$. Then, interpolating between (4.5) and (4.6) with $w^{b}$ in place of $w$, we get

$$
\left\|\Delta_{m} * H_{N+j} f\right\|_{L_{w}^{2}\left(L^{2}\right)}^{2} \leq C 2^{-\epsilon(N-m+j)}\|f\|_{L_{w}^{2}}^{2}
$$

for some $\epsilon>0$. Choose $G_{m} \in \mathcal{S}\left(\mathbb{R}^{n}\right)$ such that $\hat{G}_{m}(\xi)=F\left(A_{2^{m}}^{*} \xi\right), F \in C_{0}^{\infty}$, $\operatorname{supp}(F) \subset\left\{d_{1}<s(\xi)<d_{2}\right\}$ for some $d_{1}, d_{2}>0$, and $\Delta_{m} * G_{m} * f=\Delta_{m} * f$. Then 
the Littlewood-Paley inequality and (4.7) imply

$$
\begin{aligned}
\sum_{N \in \mathbb{Z}}\left\|\left(\delta-Q_{2^{N}}\right) * H_{N+j} f\right\|_{L_{w}^{2}\left(L^{2}\right)}^{2} & \leq C \sum_{N \in \mathbb{Z}} \sum_{m \leq N}\left\|\Delta_{m} * H_{N+j} f\right\|_{L_{w}^{2}\left(L^{2}\right)}^{2} \\
& \leq C \sum_{N \in \mathbb{Z}} \sum_{m \leq N} 2^{-\epsilon(N-m+j)}\left\|G_{m} * f\right\|_{L_{w}^{2}}^{2} \\
& \leq C \sum_{m \in \mathbb{Z}} 2^{-j \epsilon}\left\|G_{m} * f\right\|_{L_{w}^{2}}^{2} \\
& \leq C 2^{-j \epsilon}\|f\|_{L_{w}^{2}}^{2} .
\end{aligned}
$$

Interpolation between (4.4) and (4.8) implies

$$
\sum_{N}\left\|\left(\delta-Q_{2^{N}}\right) * H_{N+j} f\right\|_{L_{w}^{2}\left(L^{q}\right)}^{2} \leq C 2^{-j \epsilon}\|f\|_{L_{w}^{2}}^{2}
$$

for some $\epsilon>0$, where $q, w$ are as in Theorem 2. Since

$$
I_{j}(f) \leq\left(\sum_{N \in \mathbb{Z}}\left|\left(\delta-Q_{2^{N}}\right) * H_{N+j} f\right|^{q}\right)^{1 / q}
$$

and $q \geq 2$, by (4.3) and (4.9) we have

$$
\begin{aligned}
\|I(f)\|_{L_{w}^{2}\left(L^{q}\right)} & \leq \sum_{j=0}^{\infty}\left\|\left(\sum_{N \in \mathbb{Z}}\left\|\left(\delta-Q_{2^{N}}\right) * H_{N+j} f\right\|_{L^{q}\left(S^{n-1}\right)}^{q}\right)^{2 / q}\right\|_{L_{w}^{1}}^{1 / 2} \\
& \leq \sum_{j=0}^{\infty}\left(\sum_{N \in \mathbb{Z}}\left\|\left(\delta-Q_{2^{N}}\right) * H_{N+j} f\right\|_{L_{w}^{2}\left(L^{q}\right)}^{2}\right)^{1 / 2} \\
& \leq C \sum_{j=0}^{\infty} 2^{-j \epsilon / 2}\|f\|_{L_{w}^{2}} \leq C\|f\|_{L_{w}^{2}},
\end{aligned}
$$

where $q, w$ are as in Theorem 2. This completes the proof of Lemma 3.

Proof of (4.1). We can easily prove the pointwise inequality

$$
H_{*} f(x, \theta) \leq C H_{* *} f(x, \theta)+C M f(x, \theta)+C M f(x,-\theta) .
$$

Therefore, (4.1) follows from (2.1) and Lemma 3.

\section{Applications}

Let $K(x, y)$ be a kernel on $\mathbb{R}^{n} \times \mathbb{R}^{n}$ such that

$$
K\left(x, A_{t} y\right)=t^{-\gamma} K(x, y) \quad \text { for all } t>0 \text { and }(x, y) \in \mathbb{R}^{n} \times\left(\mathbb{R}^{n} \backslash\{0\}\right) .
$$

We assume that $K(x, y)$ is locally integrable with respect to $y$ in $\mathbb{R}^{n} \backslash\{0\}$ and

$$
\int_{a \leq r(y) \leq b} K(x, y) d y=0 \quad \text { for all } a, b, 0<a<b,
$$

for every $x \in \mathbb{R}^{n}$. We consider the singular integral

$$
T f(x)=\text { p.v. } \int K(x, y) f(x-y) d y=\lim _{\epsilon \rightarrow 0} \int_{r(y) \geq \epsilon} K(x, y) f(x-y) d y
$$


and the maximal singular integral

$$
T_{*} f(x)=\sup _{\epsilon, R>0}\left|\int_{\epsilon \leq r(y) \leq R} K(x, y) f(x-y) d y\right| .
$$

We can apply Theorems 1 and 2 to study mapping properties of $T$ and $T_{*}$.

Theorem 3. Let $\left(1 / p, 1 / q^{\prime}\right) \in \Delta_{\left(2, q_{n}(2)\right)}, q^{\prime}=q /(q-1)$. Suppose that $K(x, y)$ is odd in $y$, that is, $K(x,-y)=-K(x, y)$ for all $(x, y) \in \mathbb{R}^{n} \times\left(\mathbb{R}^{n} \backslash\{0\}\right)$, and suppose that

$$
\sup _{x \in \mathbb{R}^{n}}\left(\int_{S^{n-1}}|K(x, \theta)|^{q} d \sigma(\theta)\right)^{1 / q}=\|K\|_{L^{\infty}\left(L^{q}\right)}<\infty .
$$

Then $T_{*}$ is bounded on $L^{p}\left(\mathbb{R}^{n}\right)$.

This is an analogue for $T_{*}$ of Theorem 12 of [1] concerning $T$. See [4, 8, 9] for the case of isotropic dilation.

Theorem 4. Let $2(n-1) / n<q \leq 2, w \in \mathcal{A}_{1}$. Suppose that $\|K\|_{L^{\infty}\left(L^{q}\right)}<\infty$. Then $T$ and $T_{*}$ are bounded on $L_{w}^{2}, w \in \mathcal{A}_{1}^{\tau}, \tau=n-2(n-1) / q$.

Since $w^{b} \in \mathcal{A}_{1}$ for some $b>1$ when $w \in \mathcal{A}_{1}$, from Theorem 4 we readily obtain the following result.

Corollary 1. Suppose that $\|K\|_{L^{\infty}\left(L^{q}\right)}<\infty$ for all $q<2$. Then $T$ and $T_{*}$ are bounded on $L_{w}^{2}$ for all $w \in \mathcal{A}_{1}$.

Using this result and the extrapolation theorem of Rubio de Francia, we can obtain the $L_{w}^{p}$ boundedness of $T$ and $T_{*}$ for $w \in \mathcal{A}_{p / 2}, p \geq 2$. As in Theorem 3 , the kernels $K$ are assumed to be odd in $y$ in Theorem 4 and Corollary 1.

Proof of Theorem 3. The method of rotations of Calderón-Zygmund and Hölder's inequality imply

$$
\begin{aligned}
T_{*} f(x) & \leq C \int_{S^{n-1}}|K(x, \theta)| H_{*} f(x, \theta) d \sigma(\theta) \\
& \leq C\|K\|_{L^{\infty}\left(L^{q}\left(S^{n-1}\right)\right)}\left\|H_{*} f(x, \cdot)\right\|_{L^{q^{\prime}}} .
\end{aligned}
$$

Thus, the conclusion follows from Theorem 1.

Similarly, Theorem 4 follows from Theorem 2.

Remark 2. Introducing nonisotropic Riesz transforms as in [12, we expect that Theorems 3 and 4 extend to the case where kernels $K(x, y)$ are even in $y$ (see [3, 8, 17]). We do not pursue this here.

\section{REFERENCES}

[1] N. Bez, Mixed-norm estimates for a class of nonisotropic directional maximal operators and Hilbert transforms, J. Funct. Anal. 255 (2008), 3281-3302. MR2469023 (2010a:42061)

[2] A. P. Calderón and A. Torchinsky, Parabolic maximal functions associated with a distribution, Advances in Math. 16 (1975), 1-64. MR0417687 (54:5736)

[3] A. P. Calderón and A. Zygmund, On singular integrals, Amer. J. Math. 78 (1956), 289-309. MR0084633(18:894a)

[4] A. P. Calderón and A. Zygmund, On singular integrals with variable kernels, Appl. Anal. 7 (1978), 221-238. MR0511145 (58:23379)

[5] Lung-Kee Chen, The singular integrals related to the Calderón-Zygmund method of rotations, Appl. Anal. 30 (1988), 319-329. MR967578 (90b:42030) 
[6] Lung-Kee Chen, The maximal operators related to the Calderón-Zygmund method of rotations, Illinois J. Math. 33 (1989), 268-279. MR987823 (90e:42034)

[7] M. Christ, Estimates for the k-plane transform, Indiana Univ. Math. J. 33 (1984), 891-910. MR763948 (86k:44004)

[8] M. Christ, J. Duoandikoetxea and J. L. Rubio de Francia, Maximal operators related to the Radon transform and the Calderón-Zygmund method of rotations, Duke Math. J. 53 (1986), 189-209. MR835805 (88d:42032)

[9] M. Cowling and G. Mauceri, Inequalities for some maximal functions. I, Trans. Amer. Math. Soc. 287 (1985), 431-455. MR768718 (86a:42023)

[10] J. Duoandikoetxea and J. L. Rubio de Francia, Maximal and singular integral operators via Fourier transform estimates, Invent. Math. 84 (1986), 541-561. MR837527 (87f:42046)

[11] E. B. Fabes and N. Rivière, Singular integrals with mixed homogeneity, Studia Math. 27 (1966), 19-38. MR0209787 (35:683)

[12] E. B. Fabes and N. Rivière, Symbolic calculus of kernels with mixed homogeneity, Proceedings of Symposia in Pure Mathematics, vol. 10, Singular integrals, Providence, RI, 1967, pp. 106127. MR0236773 (38:5067)

[13] C. Fefferman and E. M. Stein, Some maximal inequalities, Amer. J. Math. 93 (1971), 107115. MR0284802(44:2026)

[14] R. Fefferman, On an operator arising in the Calderón-Zygmund method of rotations and the Bramble-Hilbert lemma, Proc. Nat. Acad. Sci. U.S.A. 80 (1983), 3877-3878. MR706542 (85g:42019)

[15] P. Gressman, $L^{p}$-improving properties of X-ray like transforms, Math. Res. Lett. 13 (2006), 10001-10017. MR2280775 (2007i:47056)

[16] P. Gressman, Sharp $L^{p}-L^{q}$ estimates for generalized $k$-plane transforms, Adv. in Math. 214 (2007), 344-365. MR2348034 (2008m:47047)

[17] A. Nagel, N. Rivière and S. Wainger, On Hilbert transforms along curves. II, Amer. J. Math. 98 (1976), 395-403. MR0450900 (56:9191b)

[18] N. Rivière, Singular integrals and multiplier operators, Ark. Mat. 9 (1971), 243-278. MR0440268 (55:13146)

[19] J. L. Rubio de Francia, F. J. Ruiz and J. L. Torrea, Calderón-Zygmund theory for operatorvalued kernels, Adv. in Math. 62 (1986), 7-48. MR859252 (88f:42035)

[20] S. Sato, Maximal functions associated with curves and the Calderón-Zygmund method of rotations, Trans. Amer. Math. Soc. 293 (1986), 799-806. MR816326 (87f:42051)

[21] S. Sato, Estimates for singular integrals along surfaces of revolution, J. Aust. Math. Soc. 86 (2009), 413-430. MR2529333 (2010h:42023)

[22] E. M. Stein and S. Wainger, Problems in harmonic analysis related to curvature, Bull. Amer. Math. Soc. 84 (1978), 1239-1295. MR.508453 (80k:42023)

Department of Mathematics, Faculty of Education, Kanazawa University, Kanazawa 920-1192, JAPAN

E-mail address: shuichi@kenroku.kanazawa-u.ac.jp 